\title{
Overlap between attention-deficit hyperactivity disorder and neurodevelopmental, externalising and internalising disorders: separating unique from general psychopathology effects
}

Ebba Du Rietz, Erik Pettersson, Isabell Brikell, Laura Ghirardi, Qi Chen, Catharina Hartman, Paul Lichtenstein, Henrik Larsson and Ralf Kuja-Halkola

\section{Background}

Although attention-deficit hyperactivity disorder (ADHD) is classified as a neurodevelopmental disorder in the latest diagnostic manuals, it shows phenotypic and genetic associations of similar magnitudes across neurodevelopmental, externalising and internalising disorders.

\section{Aims}

To investigate if $A D H D$ is aetiologically more closely related to neurodevelopmental than externalising or internalising disorder clusters, after accounting for a general psychopathology factor.

\section{Method}

Full and maternal half-sibling pairs $(N=774416)$, born between 1980 and 1995, were identified from the Swedish Medical Birth and Multi-Generation Registers, and ICD diagnoses were obtained from the Swedish National Patient Register. A higherorder confirmatory factor analytic model was fitted to examine associations between ADHD and a general psychopathology factor, as well as a neurodevelopmental, externalising and internalising subfactor. Quantitative genetic modelling was performed to estimate the extent to which genetic, shared and non-shared environmental effects influenced the associations with ADHD

\section{Results}

ADHD was significantly and strongly associated with all three factors $(r=0.67-0.75)$. However, after controlling for a general psychopathology factor, only the association between ADHD and the neurodevelopmental-specific factor remained moderately strong $(r=0.43,95 \% \mathrm{Cl}=0.42-0.45)$ and was almost entirely influenced by genetic effects. In contrast, the association between ADHD and the externalising-specific factor was smaller $(r=0.25,95 \% \mathrm{Cl}=0.24-0.27)$, and largely influenced by nonshared environmental effects. There remained no internalisingspecific factor after accounting for a general factor.

\section{Conclusions}

Findings suggest that ADHD comorbidity is largely explained by genetically influenced general psychopathology, but the strong link between ADHD and other neurodevelopmental disorders is also substantially driven by unique genetic influences.

\section{Keywords}

Aetiology; attention-deficit hyperactivity disorders; autism spectrum disorders; comorbidity; genetics.

\section{Copyright and usage}

(c) The Authors, 2020. Published by Cambridge University Press on behalf of the Royal College of Psychiatrists. This is an Open Access article, distributed under the terms of the Creative Commons Attribution licence (http://creativecommons.org/ licenses/by/4.0/), which permits unrestricted re-use, distribution, and reproduction in any medium, provided the original work is properly cited.
In the former versions of the diagnostic manuals DSM-IV-TR ${ }^{1}$ and ICD-10, ${ }^{2}$ attention-deficit hyperactivity disorder (ADHD) was classified as part of disruptive behaviour disorders, often referred as 'externalising' disorders. ${ }^{3,4}$ In the recently updated versions of the DSM-5 and ICD-11, ADHD is classified as a neurodevelopmental disorder. This change is supported by the high comorbidity and similar characteristics between ADHD and neurodevelopmental disorders. ${ }^{5-7}$ A recent meta-analysis of twin studies, however, suggested substantial genetic overlap between ADHD and not only neurodevelopmental disorders, but also externalising and internalising disorders (genetic correlations, 0.49-0.56). ${ }^{8}$ These quantitative genetic findings have been paralleled by recent molecular genetic research, further suggesting similar magnitudes of genetic associations between ADHD and neurodevelopmental, externalising and internalising disorders. ${ }^{9-11}$ The substantial degree of genetic overlap between psychiatric disorders demonstrates that genetic variants that contribute to risk for developing psychiatric disorders have highly pleiotropic effects, i.e. influencing multiple disorders. There has been an increased research interest into a latent general psychopathology factor that explains phenotypic and genetic variation (10-57\%) across psychiatric conditions, including ADHD. ${ }^{12-18}$ However, it remains unclear whether genetic effects for ADHD are shared with other neurodevelopmental disorders, as well as externalising and internalising disorders, after accounting for general psychopathology. Some evidence for genetic specificity comes from a population-based study that investigated associations between polygenic risk score (PRS) for ADHD and childhood psychiatric symptoms after controlling for a general psychopathology factor; ${ }^{15}$ the specific association between ADHD PRS and hyperactivity-impulsivity symptoms was significant, but not with other neurodevelopmental, externalising or internalising symptoms. ${ }^{15}$ This finding is largely in line with another study that used ADHD PRS in children. ${ }^{19}$ These prior studies, however, used PRSs of limited power to capture genetic variation associated with disease specificity, ${ }^{15,19}$ and have mainly focused on psychiatric symptoms obtained in childhood. Further research, using statistically powerful methods and clinical assessments beyond childhood, are therefore needed to understand if ADHD is aetiologically closer to certain psychiatric domains after accounting for general psychopathology.

\section{Aims}

The aim of this study is to use a large-scale, multivariate sibling design of children and young adults to estimate the general versus 
specific (after controlling for general effects) psychopathologic influences that explain the associations between ADHD and comorbid disorder clusters (neurodevelopmental, externalising and internalising), and to what extent these are genetic or environmental in origin. Despite uncertainties in the current research literature, we hypothesise that ADHD will be most aetiologically closely linked to the neurodevelopmental cluster after accounting for a general psychopathology factor, in light of the similar disorder characteristics, ${ }^{5-7}$ which would support the structure of the recently revised diagnostic manuals.

\section{Method}

\section{Sample}

Our source population comprised all individuals born in Sweden between 1980 and 1995, identified from the Medical Birth Register (1 688807 individuals). We excluded individuals who were born with congenital malformations $(n=80912)$, died $(n=7412)$ or emigrated $(n=57389)$ before the age of 15 years. Using Swedish personal identification numbers, we linked several nation-wide registers. The National Patient Register includes psychiatric in-patient admissions in Sweden since 1973 and out-patient diagnoses since 2001, classified according to the ICD-8 (1969-1986), ICD-9 (1987-1996) or ICD-10 (1997-present). Lifetime diagnoses were treated as binary variables (presence/absence). Each participant could receive more than one diagnosis during the study period.

Using the Multi-Generation Register, we identified all full and maternal half-siblings, excluding adopted children $(n=11266)$. Among individuals without half-siblings, we selected one random full sibling pair per family who were not twins. In families with half-siblings, we randomly selected one pair of maternal half-siblings. In total, we included 774416 individuals from 341066 full and 46142 maternal half-sibling pairs. The authors assert that all procedures contributing to this work comply with the ethical standards of the relevant national and institutional committees on human experimentation and with the Helsinki Declaration of 1975, as revised in 2008. All procedures involving human patients were approved by the Regional Ethical Review Board in Stockholm, Sweden (Dnr 2013/862-31/5). Since our study was based on population registers, the requirement for informed consent was waived.

\section{Measures}

We extracted main and secondary ICD-9 and ICD-10 diagnoses from in-patient or out-patient services from the National Patient Register. We focused on neurodevelopmental, externalising and internalising disorders that are polygenic in nature (e.g. Rett's syndrome was excluded; see Supplementary Table 1, available at https://doi.org/10. 1192/bjp.2020.152, for included disorders and ICD codes).

\section{Statistical analysis}

Phenotypic analyses

We grouped the disorders into three clusters based on the structure in the DSM-5 and ICD-11, and on clustering structures from previous literature. ${ }^{3,20-22}$ The neurodevelopmental cluster included autism spectrum disorder, developmental and learning disorders, intellectual disability and motor disorders. The externalising cluster included oppositional defiant disorder, conduct disorder, antisocial personality disorder, alcohol misuse and drug misuse. Conduct disorder, oppositional defiant disorder and antisocial personality disorders were grouped as one disorder category because of low prevalence rates and the moderately high conversion rate of oppositional defiant disorder and conduct disorder into adult antisocial personality disorder. ${ }^{23,24}$ The internalising cluster included depression, general anxiety, phobic disorders, reactions to severe stress and adjustment disorders (e.g. post-traumatic stress disorder), and obsessive-compulsive disorder.

We fitted a higher-order confirmatory factor analytic model on individual-level phenotypic data across full and maternal halfsibling pairs, hereafter referred to as the general factor model. This model was suitable for our research question, as it enables partitioning the relative contributions of general and cluster-specific factors from the overall correlations between ADHD and disorder clusters. The three latent subfactors (neurodevelopmental, externalising and internalising subfactors) were set to load onto the psychiatric disorders. We modelled a general psychopathology factor, which loaded onto each of the three subfactors. Correlations between the subfactors were fixed to zero so that they only correlated through the psychopathology factor. Each subfactor had an additional loading from a specific factor (i.e. residual variance not explained by the general factor), referred to as the neurodevelopmental-, externalising- and internalising-specific factors. Correlations between each specific factor and the general factor were fixed to zero. We then correlated $\mathrm{ADHD}$ with the general and the three specific factors.

\section{Quantitative genetic analyses}

We used a multivariate sibling design to establish the genetic and environmental aetiology of the general factor and the three subfactors (neurodevelopmental, externalising and internalising) by specifying additive genetic (A), shared environmental (C) and nonshared environmental (E) latent variables as sources of variance and covariance between factors. ${ }^{13} \mathrm{~A}$-variables were estimated by fixing them to correlate between siblings at their expected average sharing of cosegregating genes ( 0.5 for full siblings, 0.25 for half-siblings). C-variables (i.e. non-genetic components that make sibling pairs similar) were estimated by fixing them to correlate at unity across full and half-siblings. Thus, we assumed the shared environment variables to be equally shared across full and maternal halfsiblings, as previous literature has shown strong support for this assumption in Swedish registers. ${ }^{13}$ E-variables were estimated by fixing them to correlate at zero across all siblings, thus measuring non-genetic components making siblings within a pair dissimilar.

All analyses included gender and birth year (as categories, see Table 1) as covariates to adjust for different follow-up lengths and cohort effects. Analyses were performed in R (version 3.4.1 for Windows), using the 'corrplot' (version 0.84) and 'OpenMx' packages (version 2.15.5). ${ }^{25,26}$ Because half-siblings tend to display a higher rate of disorders than full siblings, ${ }^{27,28}$ prevalence rates were allowed to vary across full and half-siblings. We used weighted least squares for model fitting, and $\chi^{2}$-tests to compare nested models. We evaluated if the models provided a good fit using the root-mean-square error of approximation (RMSEA; comparison to the maximum possible fit to the data) and the comparative fit index (CFI; comparison to a model where correlations between observed variables are assumed to be zero). ${ }^{29}$ Data analysis was performed between 3 May 2019 and 14 January 2020.

\section{Secondary analyses}

To allow comparability with prior findings in the literature, we ran additional analyses with methodological approaches that have been more commonly used in research. First, we conducted univariate ACE modelling of the psychiatric disorders to allow comparability with estimates from twin studies. Second, we fitted a correlated factors model, where disorders loaded onto one of the three neurodevelopmental, externalising and internalising subfactors (allowing subfactors to correlate and without a general factor). We additionally ran a bifactor model, which derives the general psychopathology 


\begin{tabular}{|c|c|c|c|}
\hline & All, $n(\%)$ & Males, $n(\%)$ & Females, $n(\%)$ \\
\hline Total & $774416(100.0)$ & $397175(51.3)$ & $377241(48.7)$ \\
\hline Born 1980-83 & $139336(18.0)$ & 71605 (18.0) & $67731(18.0)$ \\
\hline Born 1984-87 & $195555(25.3)$ & $99962(25.2)$ & $95593(25.3)$ \\
\hline Born 1988-91 & $247113(31.9)$ & $127119(32.0)$ & $119994(31.8)$ \\
\hline Born 1992-95 & $192412(24.8)$ & $98489(24.8)$ & 93923 (24.9) \\
\hline Full siblings & $682132(88.1)$ & $350420(88.2)$ & $331712(87.9)$ \\
\hline Maternal half-siblings & 92284 (11.9) & $46755(11.8)$ & $45529(12.1)$ \\
\hline Followed until age, yr, mean (s.d.) & $25.29(4.22)$ & $25.32(4.22)$ & $25.26(4.22)$ \\
\hline Attention-deficit hyperactivity disorder & $18912(2.4)$ & $11512(2.9)$ & $7400(2.0)$ \\
\hline \multicolumn{4}{|l|}{ Neurodevelopmental disorders } \\
\hline Autism spectrum disorder & $9012(1.2)$ & $5789(1.5)$ & $3223(0.9)$ \\
\hline Developmental disorders & $3615(0.5)$ & $2267(0.6)$ & $1348(0.4)$ \\
\hline Intellectual disability & $5729(0.7)$ & $3327(0.8)$ & $2402(0.6)$ \\
\hline Motor disorders & $2663(0.3)$ & $1921(0.5)$ & $742(0.2)$ \\
\hline \multicolumn{4}{|l|}{ Externalising disorders } \\
\hline Oppositional defiant and related disorders & $2261(0.3)$ & $1414(0.4)$ & $847(0.2)$ \\
\hline Alcohol misuse & $25784(3.3)$ & $13466(3.4)$ & $12318(3.3)$ \\
\hline Drug misuse & $13458(1.7)$ & $8311(2.1)$ & 5147 (1.4) \\
\hline \multicolumn{4}{|l|}{ Internalising disorders } \\
\hline Depression & $40020(5.2)$ & $14613(3.7)$ & $25407(6.7)$ \\
\hline Generalised anxiety disorder & $36186(4.7)$ & $12568(3.2)$ & $23618(6.3)$ \\
\hline Phobias & $7700(1.0)$ & $3096(0.8)$ & $4604(1.2)$ \\
\hline Reactions to severe stress and adjustment disorders & $17661(2.3)$ & $6023(1.5)$ & $11638(3.1)$ \\
\hline Obsessive-compulsive disorder & $5724(0.7)$ & $2316(0.6)$ & $3408(0.9)$ \\
\hline
\end{tabular}

factor from the correlation matrix between the psychiatric disorders rather than higher-order subfactors. With this bifactor model, we aimed to investigate if the patterns of within-individual and between-sibling correlations of ADHD with the cluster-specific factors remained when the correlation between disorders in different clusters were not forced to correlate through the general psychopathology factor via the cluster subfactors. To examine the robustness of our findings across different sibling selection criteria, we re-ran the general factor quantitative genetic analyses on sibling pairs born closest together rather than random pairs in a family. Finally, to examine whether differences in disease onset (e.g. early versus late adult onset of the non-neurodevelopmental disorders) had a substantial confounding effect on the structure of our general factor model, and on phenotypic and aetiologic associations of disorder clusters with ADHD, we repeated the general factor quantitative genetic analyses, decreasing the maximum follow-up age by 1 year at a time. This was achieved by increasing the earliest included birth year from 1980 to 1990 by 1 year at a time, with follow-up ages ranging from $18-33$ to $18-23$ years.

\section{Results}

In the sample, $51.3 \%$ were males and the average follow-up length was until 25.3 years of age (Table 1 for information on gender, birth year and prevalence of psychiatric disorders). Figure 1 displays the within-individual phenotypic (tetrachoric) correlations between psychiatric disorders (Supplementary Table 2 for 95\% CIs). The overall pattern of correlations showed that psychiatric disorders most strongly correlated with other disorders within their respective, prespecified neurodevelopmental, externalising or internalising clusters, whereas the correlations between ADHD and other disorders were difficult to characterise.

\section{General factor model}

Phenotypic analyses

The general factor model fit the data well (RMSEA $=0.006$, $\mathrm{CFI}=0.974)$. The general psychopathology factor loaded moderately to strongly on the neurodevelopmental, externalising and internalising subfactors (factor loadings $0.60,0.77$ and 0.99 , respectively) (Supplementary Table 3a). ADHD was significantly and strongly correlated with the general psychopathology factor $(r=0.67)$. The total correlations between ADHD and the neurodevelopmental, externalising and internalising subfactors (sum of contributions through the general psychopathology and cluster-specific factors) were $0.75,0.67$ and 0.67 , respectively. Because the general psychopathology factor loaded almost perfectly on the internalising subfactor, we could not estimate valid standard errors. We therefore fixed the internalising subfactor to have a loading of 1 from the psychopathology factor, which did not lead to a worse model fit (Supplementary Table 3b) and did not affect the subsequent estimates (Supplementary Tables 3-8). In this model, the magnitude of loadings on the neurodevelopmental and externalising subfactors from the general psychopathology factor, and their correlations with ADHD, remained unchanged (Fig. 2 and Supplementary Tables $3 \mathrm{c}$ and 6 for $95 \%$ CIs).

After accounting for the general psychopathology factor, ADHD showed a significant and moderately strong phenotypic correlation with the neurodevelopmental-specific factor $(r=0.43$, $95 \% \mathrm{CI}=0.42-0.45)$, and a significantly smaller correlation with the externalising-specific factor $(r=0.25,95 \% \mathrm{CI}=0.24-0.27)$. Given that variance in the internalising factor was entirely subsumed by its covariance with the general psychopathology factor (loading fixed at 1), a correlation was not estimable with the internalising-specific factor (Fig. 2 and Supplementary Table 6).

\section{Quantitative genetic analyses}

Observed correlations among psychiatric disorders and latent factors between full and maternal half-sibling pairs are shown in Supplementary Tables 7 and 9.

The model fitting results estimated the heritability of the general psychopathology factor at 0.49 , with the contribution of shared environment at 0.07 and non-shared environment at 0.44 . For the neurodevelopmental-specific and externalising-specific factors, the estimated heritability was 0.89 and 0.80 , the shared environment contribution was 0.09 and 0.06 and the non-shared environment 


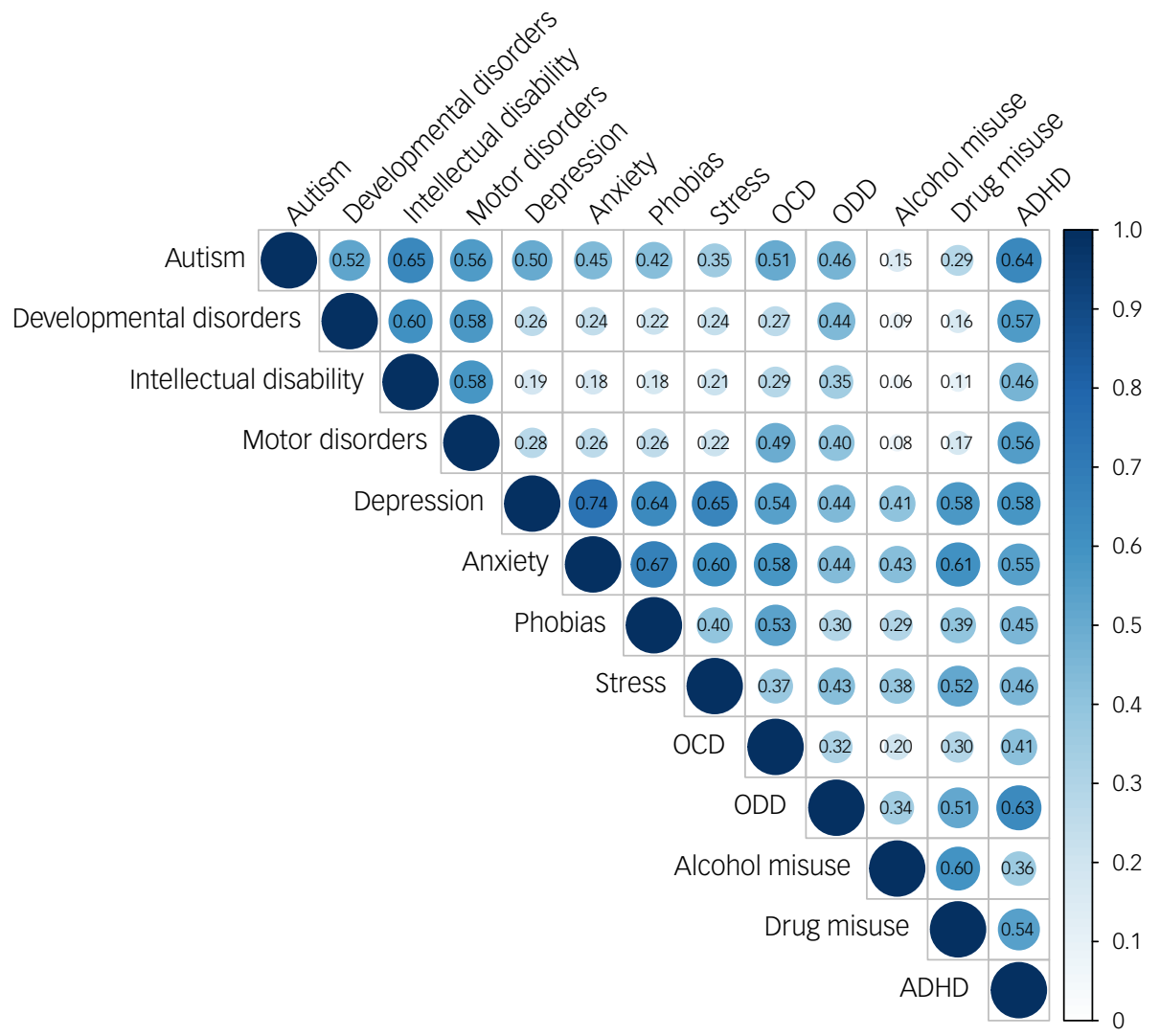

Fig. 1 Observed within-individual phenotypic correlations between disorders.

ADHD, attention-deficit hyperactivity disorder; $\mathrm{OCD}$, obsessive-compulsive disorder; ODD, oppositional defiant disorder, conduct disorder and antisocial personality disorder

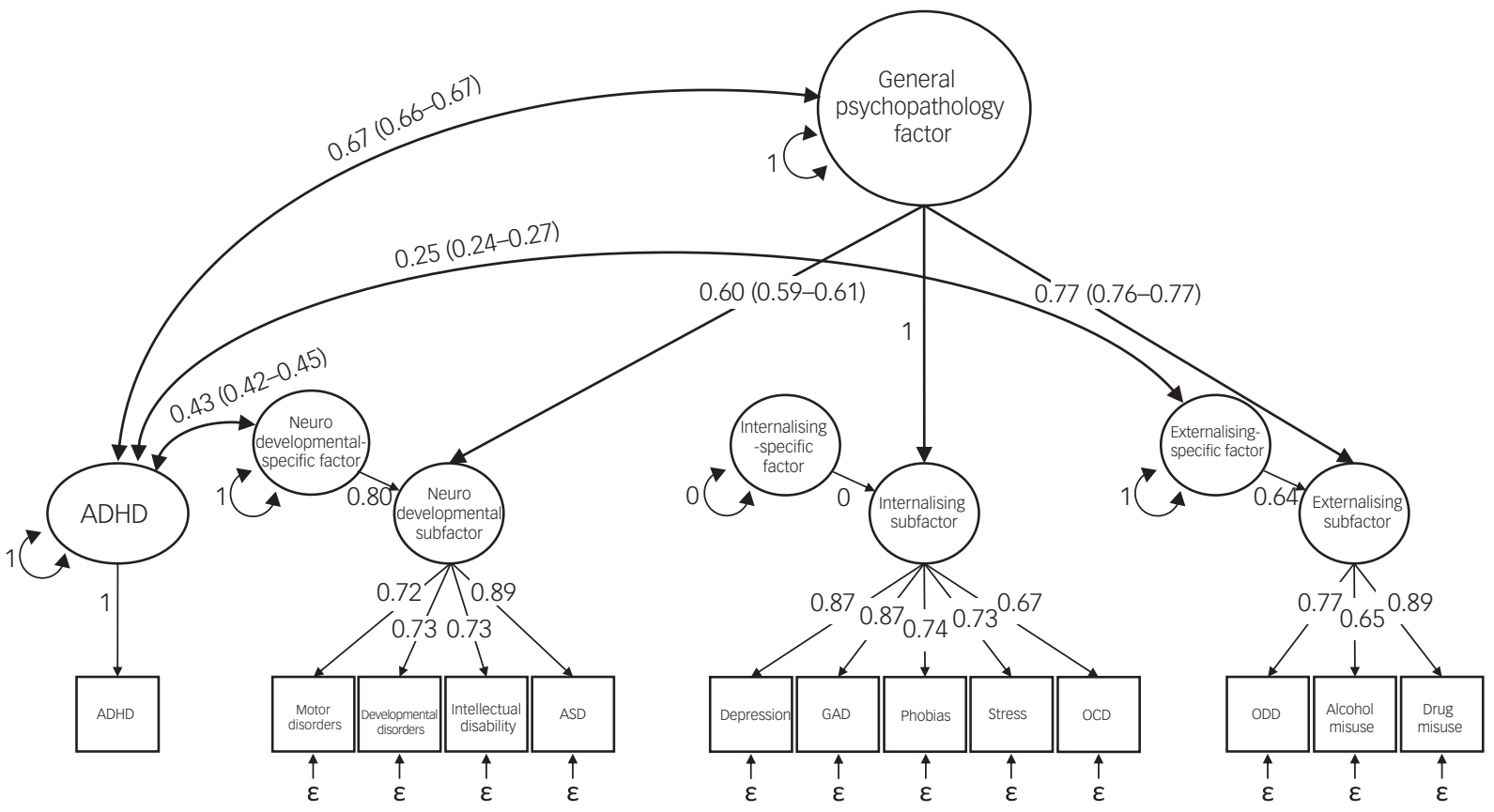

Fig. 2 General factor solution: factor loadings and phenotypic correlations between ADHD and the general psychopathology factor, neurodevelopmental-specific and externalising-specific factors.

ADHD, attention-deficit hyperactivity disorder; ASD, autism spectrum disorder; GAD, generalised anxiety disorders; OCD, obsessive-compulsive disorder; ODD, oppositional defiant, conduct and antisocial personality disorders; Stress, reactions to severe stress and adjustment disorders. 


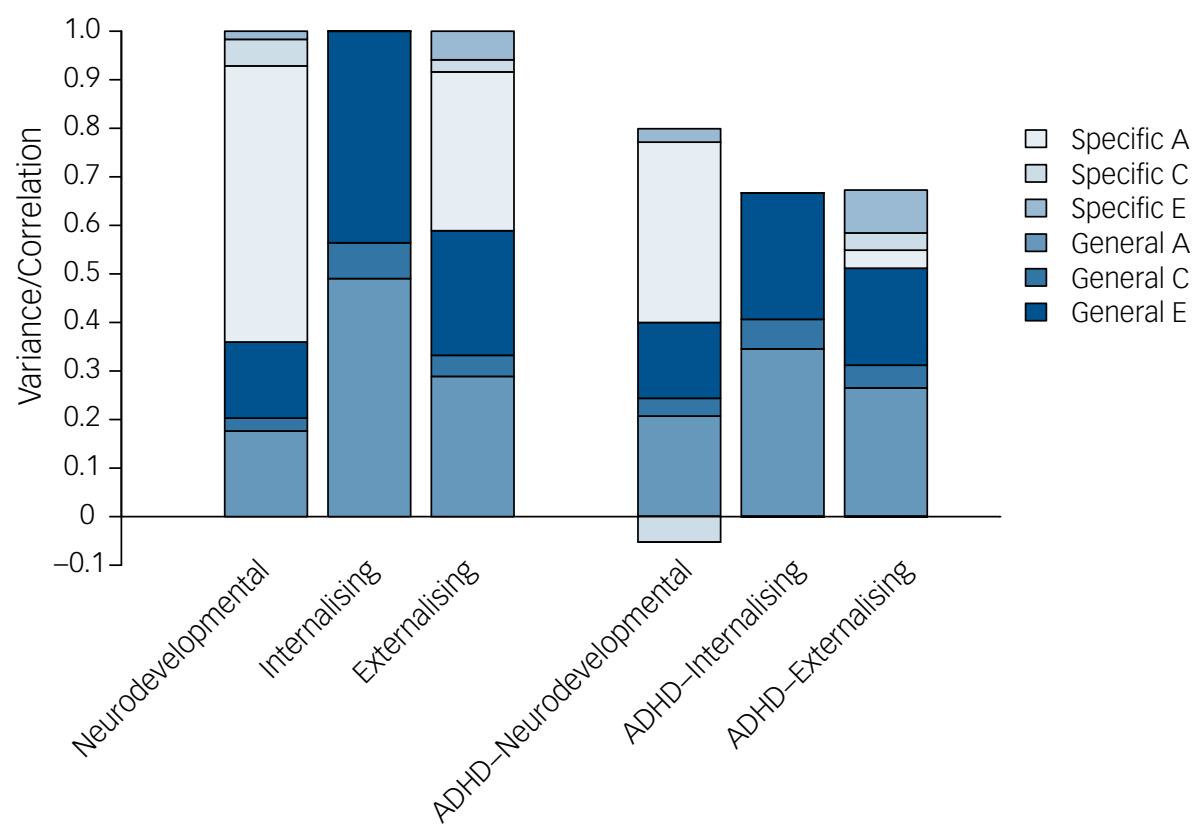

Fig. 3 General factor solution: proportion of variance in the subfactors, and in their phenotypic correlations with attention-deficit hyperactivity disorder (ADHD), explained by specific and general additive genetic (A), specific and general shared (C) and specific and general non-shared (E) environmental effects. See Supplementary Table 14 for estimates and $95 \% \mathrm{Cls}$.

contribution was 0.03 and 0.14 , respectively. Estimates are plotted in Fig. 3 and corresponding 95\% CIs are reported in Supplementary Table 10 (see Supplementary Table 11 for ACE correlations).

For the phenotypic correlation between ADHD and the general psychopathology factor $(r=0.67)$, genetics contributed with 0.35 (52\% of total correlation), shared environment contributed with $0.06(9 \%)$ and non-shared environment contributed with 0.26 (39\%) (see Supplementary Table 10 for estimates and 95\% CIs). For the phenotypic correlation between ADHD and the neurodevelopmental-specific factor $(r=0.43)$, genetics contributed with 0.46 $(107 \%$; $>100 \%$ because of negative contribution to correlation from shared environment), shared environment contributed with -0.07 ( $-15 \%$; negative contribution to correlation) and nonshared environment contributed with 0.04 (8\%). For the phenotypic correlation between ADHD and the externalising-specific factor $(r=0.25)$, genetics contributed with $0.06(23 \%)$, shared environment contributed with $0.06(22 \%)$ and non-shared environment contributed with 0.14 (55\%) (Fig. 3 and Supplementary Table 10).

\section{Secondary analyses}

See Supplementary Table 12 for univariate ACE model estimates for single disorders, which are overall in line with prior estimates from twin studies. ${ }^{30,31}$ The correlated factors model (RMSEA $=0.006$, $\mathrm{CFI}=0.974)$ revealed that the three neurodevelopmental, externalising and internalising subfactors were moderately to strongly intercorrelated $(r=0.46-0.77)$, and were all significantly and strongly correlated with ADHD $(r=0.67-0.75)$. The magnitude of correlations, along with the heritability and coheritability of the subfactors and ADHD, were overall in line with previous research (Supplementary Fig. 1). ${ }^{10,11,14,17,22}$ The bifactor model (RMSEA = $0.004, \mathrm{CFI}=0.982)$ revealed very similar within-individual and between-sibling correlations of ADHD with the general and specific factors compared with the higher-order model (Supplementary Fig. 2 and Table 13).

When we re-ran analyses on siblings born closest together, the estimates and model fit remained very similar to when we used random sibling pairs in a family (Supplementary Fig. 3). The negative contribution of the shared environment on the correlation between ADHD and the neurodevelopmental-specific factor decreased slightly in the analyses of siblings born closest together. Lastly, when analyses were repeated in subcohorts with different lengths of maximum follow-up age, the overall pattern of results remained. Although the sample size decreased with every 1-year reduction in follow-up, resulting in increased instability in estimates, the results remained similar in terms of decomposition of the correlations between ADHD and neurodevelopmental, internalising and externalising factors into specific and general $\mathrm{A}, \mathrm{C}$ and $\mathrm{E}$ components (Supplementary Fig. 4).

\section{Discussion}

In this large-scale, register-based sibling study, we showed for the first time, using clinical diagnoses, that ADHD is more phenotypically and genetically linked to neurodevelopmental disorders than to externalising and internalising disorders, after accounting for a general psychopathology factor.

The phenotypic correlations between ADHD and the neurodevelopmental, externalising and internalising subfactors were all strong, and were moderately to strongly influenced by genetic variation, in line with previous research. ${ }^{8,16,32,33}$ After accounting for the general psychopathology factor, the correlation between ADHD and the neurodevelopmental-specific factor remained moderately strong, and was largely genetic in origin, suggesting substantial unique sharing of biological mechanisms between disorders. In contrast, the correlation between ADHD and the externalising-specific factor was much smaller and was largely explained by non-shared environmental effects. The observation that there remained an association between ADHD and the externalising-specific factor after accounting for familial (genetic and shared environmental) effects is in line with a causal framework of ADHD on externalising disorders. ADHD, which often has an early onset, might subsequently 
lead to later-onset substance misuse or antisocial personality disorder. Additional research, using longitudinal causal modelling approaches, may further unravel the effect of ADHD on subsequent externalising disorders. Lastly, the correlation between ADHD and the internalising subfactor was almost entirely explained by the general psychopathology factor. This finding suggests that the comorbidity of ADHD and internalising disorders is largely because of pleiotropic genetic effects and non-shared environmental influences that have general effects on psychopathology.

\section{Research and clinical implications}

Although past research and results from our correlated factors model suggest similar magnitudes of genetic overlap between ADHD and different psychiatric disorders, we found that after accounting for general psychopathology, the strongest genetic sharing was with neurodevelopmental disorders. These findings demonstrate that isolating the psychopathology factor may allow for identifying specific mechanisms (e.g. biological pathways) and risk factors, uniquely related to only a subset of disorders or syndromes, and may have large potential for future aetiological research. For example, the Hierarchical Taxonomy of Psychopathology framework hypothesises that a higher-order approach to phenotypes may increase the precision of molecular genetic findings by differentiating between genetic liability for broad psychopathology and dimension-specific genetic risk factors. $^{34}$

Our findings on the close unique genetic link between ADHD and the neurodevelopmental subfactor are in line with the current diagnostic classification of ADHD as a neurodevelopmental disorder, despite our use of ICD codes from the former classification system. We acknowledge that there is debate regarding to what extent genetic structure of diseases should be used to inform diagnostic nosology. ${ }^{35-37}$ However, a common view in research is that genetic data, and mechanisms learned from them, can, at least in part, aid in the formulations and/or revisions of the clinical syndromes and diagnostic classification. ${ }^{37}$

\section{Limitations}

This large-scale study has several strengths, including the use of comprehensive and clinical assessments of psychiatric disorders across childhood and young adulthood, and the use of a representative population cohort. However, findings should be interpreted in light of some limitations.

Individuals with multiple psychiatric diagnoses or with siblings who have diagnoses may be more likely to get in contact with the mental health system, which can lead to an overestimation of associations among disorders and sibling pairs. On the other hand, these registers do not include individuals with relatively mild psychiatric problems, or troubled individuals who do not seek help or receive specialist care, leading to an underestimation of prevalence rates. Nevertheless, we note that our results of a general factor of psychopathology are largely consistent with previous survey studies, suggesting that results are not entirely because of these biases. ${ }^{15,16}$

During the follow-up period of individuals in our cohort, there have been changes in diagnostic practises for several psychiatric disorders (e.g. the rate of ADHD diagnoses increased fivefold from 2004 to 2015), ${ }^{38}$ and the register coverage has improved. Further, there were variations in follow-up length between individuals in our study. To account for these issues, we adjusted for the association between birth year and the expected prevalence for each disorder. However, if the change in diagnostic practises and register coverage has changed the phenotypes and aetiologies of the diagnoses, or has had differential effects on the disorders, we may have remaining bias in our results. Replication using other data sources, or similar sources with longer follow-up, would strengthen the inferences from the current study. To further account for variations in follow-up length, we also repeated analyses in younger adult subcohorts (i.e. with narrower age ranges and follow-up length), by excluding one birth year cohort at a time, starting with the oldest; findings revealed consistent patterns across these subcohorts. For the ACE models, we assumed that full and maternal half-siblings share their common (family) environment to the same degree. We acknowledge that this is a simplification, but sensitivity analyses in a similar study showed strong support for this assumption. ${ }^{13}$ Further, when we re-ran analyses with siblings born closest together (i.e. siblings who are closer in age and may therefore share more common environment), our overall results remained unchanged.

A limitation of our analysis of nation-wide administrative patient data is that findings cannot be interpreted in relation to more refined ADHD subtypes. Thus, it is important to note that there may be heterogeneity in the aetiology of ADHD, and individuals with ADHD may display different patterns of comorbidities. However, investigations into this is beyond the scope of our study and level of analysis.

Lastly, our finding that the general psychopathology factor loaded nearly perfectly on the internalising subfactor has been reported in previous research, ${ }^{16}$ and studies have often reported that internalising disorders have the highest, or among the highest, loadings from the general factor. ${ }^{12,14,17,39}$ However, it is possible that this high loading may have partly resulted from the predefined confirmatory approach to factor definitions. Furthermore, the chosen disorders in a study dictates what the psychopathology factor captures; thus, the resulting structure will be partly specific to the current study design. The bifactor model produced very similar within-individual and between-sibling correlations between $\mathrm{ADHD}$ and the neurodevelopmental, externalising and internalising subfactors compared with the higher-order model. This supports the robustness of our results, and suggests that the almost perfect loading of the general psychopathology factor on the internalising subfactor is not a major source of bias.

In conclusion, ADHD comorbidity is largely explained by partly genetically influenced general psychopathology, but the strong link between ADHD and other neurodevelopmental disorders is also driven by specific genetic influences. These findings support the classification of ADHD as a neurodevelopmental disorder in the recently revised diagnostic manuals, and provide insights into the structure of the aetiologic underpinnings of psychopathology.

\section{Ebba Du Rietz (D), PhD, Department of Medical Epidemiology and Biostatistics, Karolinska Institutet, Sweden; Erik Pettersson, PhD, Department of Medical Epidemiology and Biostatistics, Karolinska Institutet, Sweden; Isabell Brikell, PhD, The National Centre for Register-based Research, Department of Economics and Business Economics, Business and Social Science, Aarhus University, Denmark; Laura Ghirardi, PhD, Department of Medical Epidemiology and Biostatistics, Karolinska Institutet, Sweden; Qi Chen, PhD, Department of Medical Epidemiology and Biostatistics, Karolinska Institutet, Sweden; Catharina Hartman, PhD, Department of Psychiatry, University of Groningen, University Medical Center Groningen, the Netherlands; Paul Lichtenstein, PhD, Department of Medical Epidemiology and Biostatistics, Karolinska Institutet, Sweden; Henrik Larsson, PhD, School of Medical Sciences, Örebro University; and Department of Medical Epidemiology and Biostatistics, Karolinska Institutet, Sweden; Ralf Kuja-Halkola, PhD, Department of Medical Epidemiology and Biostatistics, Karolinska Institutet, Sweden}

Correspondence: Ebba Du Rietz. Email: ebba.du.rietz@ki.se

First received 23 Apr 2020, final revision 3 Jul 2020, accepted 24 Jul 2020

\section{Supplementary material}

Supplementary material is available online at https://doi.org/10.1192/bjp.2020.152 


\section{Data availability}

Data cannot be shared publicly because of the Swedish Secrecy Act. Data from the Medical Birth Register, the Multi-Generation Register and the National Patient Register were used for this study and made available by ethical approval. Researchers may apply for access through the Swedish Research Ethics Boards (www.etikprovningsmyndigheten.se) and from the primary data owners Statistics Sweden (www.scb.se) and the National Board of Health and Welfare (www.socialstyrelsen.se), in accordance with Swedish law.

\section{Author contributions}

E.D.R., H.L. and R.K.-H. were responsible for the study concept and design. E.D.R. and R.K.-H. were responsible for statistical analysis. E.D.R., E.P., I.B., L.G., Q.C., C.H., P.L., H.L. and R.K.-H. analysed and/or interpreted the data. E.D.R. and R.K.-H. drafted the manuscript E.D.R., E.P. I.B., L.G., Q.C., C.H., P.L., H.L. and R.K.-H. provided critical revision of the manuscript for important intellectual content

\section{Funding}

H.L. acknowledges financial support from the Swedish Research Council (2018-02599) and the Swedish Brain Foundation (FO2018-0273). E.D.R. was supported by grant 2019-01172 from the Swedish Research Council for Health, Working Life, and Welfare. E.P. was supported by grant 2017-01358 from the Swedish Research Council. This project has received funding from the European Union's Horizon 2020 research and innovation programme under grant agreement no. 667302 .

\section{Declaration of interest}

H.L. reported receiving grants from Shire Pharmaceuticals during the conduct of the study: personal fees from and serving as a speaker for Shire Pharmaceuticals and Evolan Pharma AB outside the submitted work; and sponsorship for a conference on attention-deficit hyperactivity side the submitted work; and sponsorship for a conference on attention-deficit hyperactivity
disorder from Shire Pharmaceuticals outside the submitted work. No other authors report any conflicts of interest.

ICMJE forms are in the supplementary material, available online at https://doi.org/10.1192/ bjp.2020.152

\section{References}

1 American Psychiatric Association. Diagnostic and Statistical Manual of Mental Disorders (4th edn, Text Revision). American Psychiatric Publishing, 2000.

2 World Health Organization. The ICD-10 Classification of Mental and Behavioural Disorders: Clinical Descriptions and Diagnostic Guidelines. World Health Organization, 1992.

3 Lahey BB, Krueger RF, Rathouz PJ, Waldman ID, Zald DH. A hierarchical causal taxonomy of psychopathology across the life span. Psychol Bull 2017; 143(2): 142-86.

4 McMahon RJ. Diagnosis, assessment, and treatment of externalizing problems in children: the role of longitudinal data. J Consult Clin Psychol 1994; 62(5): 901-17.

5 Antshel KM, Zhang-James Y, Faraone SV. The comorbidity of ADHD and autism spectrum disorder. Expert Rev Neurother 2013; 13: 1117-28.

6 Rommelse NN, Geurts HM, Franke B, Buitelaar JK, Hartman CA. A review on cognitive and brain endophenotypes that may be common in autism spectrum disorder and attention-deficit/hyperactivity disorder and facilitate the search for pleiotropic genes. Neurosci Biobehav Rev 2011; 35(6): 1363-96.

7 Doernberg E, Hollander E. Neurodevelopmental disorders (ASD and ADHD): DSM-5, ICD-10, and ICD-11. CNS Spectrums 2016; 21: 295-9.

8 Andersson A, Tuvblad A, Chen Q, Du Rietz E, Cortese S, Kuja-Halkola R, et al. The strength of the genetic overlap between ADHD and other psychiatric symptoms: a systematic review and meta-analysis. To be published in J Child Psychol Psychiatry [Preprint] 2020. Available from: https://doi.org/10.1111/ jcpp.13233 [cited 20 Mar 2020].

9 Demontis D, Walters RK, Martin J, Mattheisen M, Als TD, Agerbo E, et al. Discovery of the first genome-wide significant risk loci for attention deficit/ hyperactivity disorder. Nat Genet 2019; 51(1): 63-75.

10 Cross-Disorder Group of the Psychiatric Genomics Consortium. Genomic relationships, novel loci, and pleiotropic mechanisms across eight psychiatric disorders. Cell 2019; 179(7): 1469-82.

11 Du Rietz E, Coleman J, Glanville K, Choi SW, O'Reilly PF, Kuntsi J. Association of polygenic risk for attention-deficit/hyperactivity disorder with co-occurring traits and disorders. Biol Psychiatry Cogn Neurosci Neuroimaging 2018; 3(7): 635-43.

12 Caspi A, Houts RM, Belsky DW, Goldman-Mellor SJ, Harrington H, Israel S, et al. The P factor: one general psychopathology factor in the structure of psychiatric disorders? Clin Psychol Sci 2014; 2: 119-37.
13 Pettersson $\mathrm{E}$, Larsson $\mathrm{H}$, Lichtenstein $\mathrm{P}$. Common psychiatric disorders share the same genetic origin: a multivariate sibling study of the Swedish population. Mol Psychiatry 2016; 21(5): 717-21.

14 Sezlam S, Coleman JRI, Caspi A, Moffitt TE, Plomin R. A polygenic $P$ factor for major psychiatric disorders. Transl Psychiatr 2018; 8(1): 205.

15 Brikell I, Larsson H, Lu Y, Pettersson E, Chen Q, Kuja-Halkola R, et al. The contribution of common genetic risk variants for ADHD to a general factor of childhood psychopathology. Mol Psychiatry 2020; 25: 1809-21.

16 Lahey BB, van Hulle CA, Singh AL, Waldman I, Rathouz PJ. Higher-order genetic and environmental structure of prevalent forms of child and adolescent psychopathology. Arch Gen Psychiatry 2011; 68(2): 181-9.

17 Waldman ID, Poore HE, van Hulle C, Rathouz PJ, Lahey BB. External validity of a hierarchical dimensional model of child and adolescent psychopathology: tests using confirmatory factor analyses and multivariate behavior genetic analyses. J Abnorm Psychol 2016; 125: 1053-66.

18 Allegrini A, Cheesman R, Rimfeld K, Selzam S, Pingault JB, Eley TC, et al. The P factor: genetic analyses support a general dimension of psychopathology in childhood and adolescence. J Child Psychol Psychiatry 2019; 61(1): 30-9.

19 Riglin L, Thapar AK, Leppert B, Martin J, Richards A, Anney R, et al. Using genetics to examine a general liability to childhood psychopathology. Behav Genet 2020; 50: 213-20.

20 Kendler KS, Prescott CA, Myers J, Neale MC. The structure of genetic and environmental risk factors for common psychiatric and substance use disorders in men and women. Arch Gen Psychiatry 2003; 60(9): 929-37.

21 Luningham JM, Poore HE, Yang J, Waldman ID. Testing structural models of psychopathology at the genomic level. BioRxiv [Preprint] 2019. Available from: https://doi.org/10.1101/502039 [cited 10 Mar 2020].

22 Krueger RF. The structure of common mental disorders. Arch Gen Psychiatry 1999; 56(10): 921-6.

23 Moffitt TE, Arseneault L, Jaffee SR, Kim-Cohen J, Koenen KC, Odgers CL, et al. Research review: DSM-V conduct disorder: research needs for an evidence base. J Child Psychol Psychiatry 2008; 49(1): 3.

24 Simonoff E, Elander J, Holmshaw J, Pickles A, Murray R, Rutter M. Predictors of antisocial personality disorder. Continuities from childhood to adult life. $\mathrm{Br} J$ Psychiatry 2004; 184: 118.

25 Neale MC, Hunter MD, Pritikin JN, Zahery M, Brick TR, Kirkpatrick RM, et al. OpenMx 2.0: extended structural equation and statistical modeling. Psychometrika 2016; 81(2): 535-49.

26 Wei T, Simko V. R Package "corrplot": Visualization of a Correlation Matrix (Version 0.84). GitHub, 2017 (https://github.com/taiyun/corrplot).

27 Kuja-Halkola R, D'Onofrio BM, Larsson $\mathrm{H}$, Lichtenstein P. Maternal smoking during pregnancy and adverse outcomes in offspring: genetic and environmental sources of covariance. Behav Genet 2014; 44(5): 456-67.

28 Yao S, Kuja-Halkola R, Martin J, Lu Y, Lichtenstein P, Norring C, et al. Associations between attention-deficit/hyperactivity disorder and various eating disorders: a Swedish nationwide population study using multiple genetically informative approaches. Biol Psychiatry 2019; 86(8): 577-86.

29 Hu LT, Bentler PM. Cutoff criteria for fit indexes in covariance structure anaIysis: conventional criteria versus new alternatives. Struct Equ Modeling 1999; 6: 1-55.

30 Larsson $\mathrm{H}$, Chang Z, D'Onofrio BM, Lichtenstein P. The heritability of clinically diagnosed attention deficit hyperactivity disorder across the lifespan. Psychol Med 2014; 44: 2223-9.

31 Polderman TJ, Benyamin B, de Leeuw CA, Sullivan PF, van Bochoven A Visscher PM, et al. Meta-analysis of the heritability of human traits based on fifty years of twin studies. Nat Genet 2015; 47(7): 702-9.

32 Kessler RC, Adler L, Barkley R, Biederman J, Conners CK, Demler O, et al. The prevalence and correlates of adult ADHD in the United States: results from the National Comorbidity Survey Replication. Am J Psychiatry 2006; 163: 716-23.

33 Kessler RC, Chiu WT, Demler O, Walters EE. Prevalence, severity, and comorbidity of 12-month DSM-IV disorders in the National Comorbidity Survey Replication. Arch Gen Psychiatry 2005; 62: 617-27.

34 Waszczuk MA, Eaton NR, Krueger RF, Shackman AJ, Waldman ID, Zald DH, et al. Redefining phenotypes to advance psychiatric genetics: implications from hierarchical taxonomy of psychopathology. J Abnorm Psychol 2020; 129: 143-61.

35 Hyman SE. The diagnosis of mental disorders: the problem of reification. Annu Rev Clin Psychol 2010; 17: 11-21.

36 Insel TR, Cuthbert B, Garvey M, Heinssen R, Pine DS, Quinn K, et al. Research domain criteria (RDOC): toward a new classification framework for research on mental disorders. Am J Psychiatry 2010; 167: 748-51.

37 Smoller JW, Andreassen OA, Edenberg HJ, Farone SV, Glatt SJ, Kendler KS. Psychiatric genetics and the structure of psychopathology. Mol Psychiatry 2019: 24(3): 409-20. 
38 Rydell M, Lundström S, Gillberg C, Lichtenstein P, Larsson J. Has the attention deficit hyperactivity disorder phenotype become more common in children between 2004 and 2014? Trends over 10 years from a Swedish general population sample. J Child Psychol Psychiatry 2018; 59(8): 863-71.

39 Gard AM, Ware EB, Hyde LW, Schmitz L, Faul J, Mitchell C. Assessing the structure and specificity of polygenic scores for psychiatric disorders in a population-based cohort of older adults. BioRxiv [Preprint] 2019. Available from: https://doi.org/10.1101/601609 [cited 15 Mar 2020]. psychiatry in history

\title{
Aretaeus the Cappadocian advanced empirical psychiatric nosology on a biological basis, acknowledged the limits of his treatments and knew the dual powers of love
}

\author{
Greg Wilkinson (iD
}

Aretaeus of Cappadocia ( 2nd century AD), the celebrated Greek physician, is reputedly second only to Hippocrates in clinical observation. He is credited with the first description of coeliac disease, the naming and earliest accurate account of diabetes, the first correct depiction of asthma - among other things. Phrenitis, hysteria, melancholy and mania were identified before him but Aretaeus advanced empirical psychiatric nosology on a contemporaneous biological basis.

He was forgotten until the discovery in 1552 of two incomplete manuscripts on the causes, symptoms and treatment of acute and chronic diseases. A Latin translation appeared in Venice and 2 years later the original Greek text was published in Paris. In English, he is best known from a translation (1856) by Francis Adams (1796-1861), an overlooked medical scholar and the family doctor for 42 years in rural Banchory, Aberdeenshire.

The Cappadocian tells that phrenitis is seated in the head and senses. There is no surviving explanation of and treatment for hysterical suffocation, perhaps because missing or of spontaneous resolution. Melancholy is lowness of spirits from a single phantasy: the commencement and a part of mania. There is not one particular form of melancholy, which with treatment is entirely removed or has intervals of several years, but generally melancholy is again engendered. Mania is madness, derangement of mind: one genus and infinite in species. Those who have formerly been mad are more prone to melancholy. Dotage in old age never intermits, until death, whereas mania intermits and, with care, ceases altogether; but if patients attain some relaxation from the condition, they become torpid, dull, sorrowful; for having come to a knowledge of the disease they are saddened with their own calamity.

Against that background, Aretaeus says:

"It is impossible, indeed, to make all the sick well, for a physician would thus be superior to a god; but the physician can produce respite from pain, intervals in diseases, and render them latent:'

yet,

'A story is told, that a certain person, incurably affected, fell in love with a girl; and when the physicians could bring him no relief, love cured him. But I think that he was originally in love, and that he was dejected and spiritless from being unsuccessful with the girl, and appeared to the common people to be melancholic. He then did not know that it was love; but when he imparted the love to the girl, he ceased from his dejection, and dispelled his passion and sorrow; and with joy he awoke from his lowness of spirits, and he became restored to understanding, love being his physician.'

Although some advocate that Aretaeus plagiarised Archigenes of Apamea. That is another story.

(c) The Authors, 2020. Published by Cambridge University Press on behalf of the Royal College of Psychiatrists 